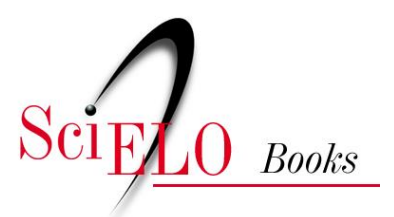

Fiockuz

ipea

\title{
Esboços para um cenário das condições de saúde da população brasileira 2022/2030
}

\author{
Mauricio L. Barreto
}

\section{SciELO Books / SciELO Livros / SciELO Libros}

BARRETO, ML. Esboços para um cenário das condições de saúde da população brasileira 2022/2030. In FUNDAÇÃO OSWALDO CRUZ. A saúde no Brasil em 2030 - prospecção estratégica do sistema de saúde brasileiro: população e perfil sanitário [online]. Rio de Janeiro:

Fiocruz/Ipea/Ministério da Saúde/Secretaria de Assuntos Estratégicos da Presidência da República, 2013. Vol. 2. pp. 97-120. ISBN 978-85-8110-016-6. Available from SciELO Books <http://books.scielo.org>.

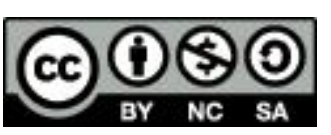

All the contents of this chapter, except where otherwise noted, is licensed under a Creative Commons Attribution-Non Commercial-ShareAlike 3.0 Unported.

Todo o conteúdo deste capítulo, exceto quando houver ressalva, é publicado sob a licença Creative Commons Atribuição Uso Não Comercial - Partilha nos Mesmos Termos 3.0 Não adaptada.

Todo el contenido de este capítulo, excepto donde se indique lo contrario, está bajo licencia de la licencia Creative Commons Reconocimento-NoComercial-CompartirIgual 3.0 Unported. 
Esboços para um Cenário das Condições de Saúde da População Brasileira 2022/2030 


\section{ESBOÇOS PARA UM CENÁRIO DAS CONDIÇÕES DE SAÚDE DA POPULAÇÃO BRASILEIRA 2022/2030}

Mauricio L. Barreto

\section{1 | INTRODUÇÃO}

No decorrer do século XX o Brasil experimentou intensas transformações na sua estrutura populacional e no padrão de morbimortalidade. A partir da segunda metade daquele século a constante queda da taxa de natalidade, mais acentuada que a verificada nas taxas de mortalidade, tem provocado diminuição das taxas de crescimento populacional (MINISTÉRIO DA SAÚDE, 2004). Paralelamente, tem-se verificado aumento da expectativa de vida ao nascer, que passou de 45,9 anos em 1950 para 73,0 anos em 2008 (http://tabnet.datasus.gov.br/cgi/idb2009/a11tb.htm), refletindo o processo de envelhecimento da população, com aumentos contínuos e significativos da população de idosos.

As análises da situação de saúde da população brasileira identificam avanços importantes traduzidos em reduções significativas de grupos problemas. Ao mesmo tempo, com relação a outros problemas identificam reduções menos significativas, estabilidade ou ainda tendência ao crescimento. (BARRETO \& CARMO, 1994; BARRETO et al, 1997; BARRETO \& CARMO, 1998; BARRETO \& CARMO, 2000; CARMO et al., 2003; MINISTÉRIO DA SAÚDE, 2004; MINISTÉRIO DA SAÚDE, 2005; MINISTÉRIO DA SAÚDE, 2006; VICTORA et al., 2011; BARRETO et al., 2011; SCHMIDT et al., 2011, REICHENHEIM et al., 2011). Algumas vezes as tendências observadas frustram as expectativas e previsões anteriormente feitas, as quais indicavam curso diferente dos eventos.

Como exemplos de sucesso podem-se destacar as reduções observadas nas taxas de mortalidade infantil (TMI), na taxa de desnutrição em crianças e a ocorrência das doenças imunopreveníveis. A redução da TMI, intensificada a partir da década de 1960, quando apresentava uma média nacional de 117,0/1000 nascidos vivos, decresceu para a faixa de 50 por 1000 nascidos vivos ao final da década de 1980. 
No decorrer da década de 1990 registrou-se redução média nacional de mais de 40\% (49,4/1000 nascidos vivos em 1990 para 26.8/1000 nascidos vivos em 2000), chegando a 20,0/1000 nascidos vivos em 2008. A análise das tendências dos dois componentes da taxa de mortalidade infantil (neonatal e pós-neonatal) evidencia que a redução foi mais acentuada para a mortalidade infantil pós-neonatal. Este componente associase mais fortemente com fatores relacionados ao ambiente, concentrando uma maior proporção de óbitos por doenças infecciosas, particularmente as infecções intestinais. Como consequência desta redução diferencial, a mortalidade neonatal, que se relaciona mais fortemente à assistência pré e pós-natal, vem apresentando taxas mais altas do que a mortalidade pós-neonatal. Em 2007, os óbitos neonatal precoces $(<6$ dias), neonatal tardios (7 a 28 dias) e pós-neonatais (28 dias a 1 ano), representaram, respectivamente, 52,0\%, 16,5\% e 30,5\% dos óbitos de menores de um ano, no país.

A prevalência da desnutrição na população de menores de cinco anos (medida pelo déficit de altura, definido como altura por idade abaixo de -2 escores-z do padrão da Organização Mundial da Saúde) caiu de 37,1\% em 1974-5 para 7,1\% em 2006-7. Para que reduções de tais magnitudes pudessem ocorrer, reduções de ainda maior magnitude ocorreram nos grupos de mais baixa renda e na Região Nordeste. (MONTEIRO et al. 2009; VICTORA et al. 2011)

Graças a um eficiente programa de imunização, associado a um programa de fortalecimento da produção nacional de vacinas básicas, varias doenças do grupo das imunopreveníveis estão em avançado estágio de controle e para algumas delas (p.ex. sarampo e poliomielite) por muitos anos não se observam casos autóctones no país. No caso do sarampo, alguns poucos e pequenos brotes ocorridos, em período recente, no país, deveu-se a casos índices provenientes da Europa.

\section{DOENÇAS INFECCIOSAS}

Apesar da redução significativa da participação desse grupo de doenças no perfil da mortalidade do país, estas ainda têm impacto importante sobre a morbidade, como visto nos indicadores de morbidade hospitalar e no potencial de algumas destas doenças manifestarem-se sobre a forma de epidemias. Universalmente, em especial nos países desenvolvidos, a perda de importância relativa das doenças transmissíveis, ocorrida principalmente no decorrer do terceiro quarto do século XX, criou, em alguns segmentos da comunidade científica e na opinião pública, a falsa expectativa que as doenças desse grupo estariam próximas a controle ou mesmo à erradicação. Entretanto, essa crença e suas auspiciosas previsões não se confirmaram, nem mesmo para os países desenvolvidos. Essa surpreendente quebra de expectativas deveu-se ao surgimento de DIs até então não conhecidas, como a AIDS, pelo ressurgimento de doenças antigas como a cólera ou a dengue, ou pela persistência de endemias importantes, como a 
tuberculose e a hanseníase, as quais se esperavam estar controladas. Acrescente-se a isto o fato de que algumas DIs são responsáveis por situações de emergências de saúde pública em nível global, regional ou local, dado o seu potencial epidêmico, como a dengue, a SARS e a influenza por novos subtipos virais.

No tocante a sua ocorrência nas duas últimas décadas, a situação das DIs no Brasil configura um quadro complexo, que pode ser resumido em três grandes tendências: doenças transmissíveis com tendência declinante, com resultado do sucesso das medidas de controle; doenças transmissíveis com quadro de persistência, em que as medidas de controle apesar de alguma efetividade não são suficientes para controlá-las; e doenças transmissíveis em que não há sucesso no controle, inclusive algumas delas estão relacionadas a emergências de saúde pública (BARRETO et al. 2011).

Em um grande número de DIs para as quais se dispõe de instrumentos eficazes de prevenção e controle, o Brasil tem colecionado êxitos importantes. Acima já referimos ao sucesso do controle de das doenças imunopreveníveis. A varíola foi erradicada em 1973, a circulação do vírus selvagem da poliomielite cessou em 1989 e o sarampo encontra-se com a transmissão autóctone interrompida desde o ano 2000. O tétano neonatal encontra-se com taxa de incidência muito abaixo do patamar estabelecido para considerá-lo eliminado enquanto problema de saúde pública (1 por 1000 nascidos vivos). Outras doenças para as quais é possível prever uma próxima eliminação são representadas pela raiva humana transmitida por animais domésticos, peste, filariose e a síndrome da rubéola congênita. Ainda dentro deste grupo de DIs com tendência declinante, estão: a) a difteria, a coqueluche e o tétano acidental, que têm em comum o fato de serem imunopreveníveis; b) a doença de Chagas por transmissão vetorial e transfusional; c) a febre tifóide, doença que tem sua ocorrência fortemente associada com precárias condições sanitárias.

Um conjunto importante das DIs apresenta quadro de persistência, em virtude de terem sua ocorrência estabilizada ou com reduções modestas em período recente, configurando parte da agenda inconclusa nessa área, já que as medidas de controle implementadas não têm sido suficientes para controlá-las. Neste grupo destacam-se as hepatites virais - especialmente as hepatites B e C -, a esquistossomose, as leishmanioses, a hanseníase e a tuberculose, em função das altas prevalências alcançadas, da ampla distribuição geográfica em todas as regiões e do seu potencial evolutivo para formas graves que podem levar ao óbito. A malária, concentrada na Região Amazônica, onde ocorrem mais de 99\% dos casos registrados no país, até recentemente apresentava níveis persistentemente elevados, passou a apresentar tendência a redução a partir de 1999, sendo em 2002 detectados 350 mil casos, patamar que não era atingido desde o início dos anos 1980. A descontinuidade das ações de controle em muitos municípios interrompeu esta tendência declinante que somente foi retomada entre 2005 e 2006 (ano em que se registrou 540 mil casos), 
chegando, em anos recentes, para níveis em torno de 300 mil casos anuais (http:// portal.saude.gov.br/portal/saude/profissional/area.cfm?id_area=1526).

A AIDS, apesar dos esforços para controlá-la e do pioneirismo do programa brasileiro de acesso gratuito a retrovirais para todos aqueles que necessitem, pode ser também localizada no grupo de sucessos parciais. A partir da detecção do primeiro caso da AIDS no Brasil, em 1980, observou-se crescimento acelerado até 1997, ano em que foram registrados 23.545 casos novos o que significou uma incidência de 14,8 casos/100.000 hab. Até o momento foram identificados mais de 600 mil casos da doença (http://www.aids.gov.br/pagina/aids-no-brasil). Forte mobilização de setores da sociedade e o desenvolvimento de medicamentos eficazes contra o HIV vêm possibilitando a redução na intensidade de transmissão, na mortalidade e em outros danos potenciais causados por esta doença. Medidas de prevenção, como os testes rápidos para diagnostico da infecção em mulheres grávidas e o estimulo a pratica do sexo seguro, têm contribuído para redução da transmissão.

O terceiro grupo de DIs agrega aquelas doenças em que as medidas de controle não têm resultado em sucesso. Algumas delas, inclusive, com potencial para gerar situações de emergência de saúde pública, pela possibilidade de se apresentar na forma de surtos e epidemias. (BARRETO et al., 2006). Neste grupo destaca-se a dengue que tem sido objeto de um dos maiores programas de controle realizados no país e tem mobilizado o interesse do público e da mídia. As dificuldades para a eliminação de um mosquito domiciliado e que facilmente se multiplica em diferentes recipientes que armazenam água, particularmente aqueles encontrados nos lixos das cidades como garrafas, latas e pneus, ou mesmo no interior dos domicílios, como em vasos de plantas, têm exigido um esforço substancial do setor saúde. Entretanto, esse esforço necessita ser articulado com outras políticas públicas como a limpeza urbana, além de exigir a conscientização e mobilização social das comunidades sobre a necessidade de realizarem esforços para manter seu entrono livre do mosquito e das condições que estimulem sua proliferação. Nos últimos anos, vem sendo registrado aumento no número de casos. No ano 2010, alcançou cerca de 1,4 milhões de casos com 95 mil hospitalizações e 300 óbitos. A circulação sequencial de mais de um sorotipo, além de outros mecanismos não conhecidos, vem propiciando o aumento na incidência de febre hemorrágica da dengue, com consequente incremento de casos severos, que necessitam imediata hospitalização, e dos óbitos por esta doença. A introdução do sorotipo DEN4 em 2010 representa um fator potencial que favorece uma nova onda epidêmica do dengue, como aumento das possibilidades de crescimento da ocorrência de formas hemorrágicas e outras formas severas. 


\section{3 | DOENÇAS CRÔNICAS NÃO TRANSMISSÍVEIS}

As Doenças Crônicas não Transmissíveis (DCNTs), em especial nos países em desenvolvimento, vem exercendo papel cada vez mais importante no perfil de saúde das populações humanas. Não por acaso, em 2011 as Nações Unidas convocaram reunião de chefes de Estados para avaliar a questão e definir ações globais para reduzir os seus efeitos. Estimativas da Organização Mundial de Saúde (OMS) apontam que as DCNTs já são responsáveis por 58,5\% de todas as mortes ocorridas no mundo e por 45,9\% da carga global de doença (WHO, 2002). No Brasil, em 2007, as DCNTs respondiam por cerca $72,0 \%$ do total das mortes por causas conhecidas (SCHMIDT et. al., 2011). Séries históricas de estatísticas de mortalidade disponíveis para as capitais dos estados brasileiros indicam que a proporção de mortes por DCNTs aumentou em mais de três vezes entre as décadas de 30 e de 90 (MALTA, et al., 2007a; MOURA, et al; 2008). Dentre elas as doenças do aparelho circulatório passaram de 10\% do total de óbitos na década de 1930 para cerca de 30\% em 2005, as neoplasias passaram de 2\% para 13,6\% dos óbitos, no mesmo período (MINISTÉRIO DA SAÚDE, 2006).

Dentre as DCNTs destacam-se as doenças do aparelho circulatório, que representam a principal causa de morte, em ambos os sexos. Dentre estas, as doenças cerebrovasculares constituem a causa mais frequente e as doenças isquêmicas do coração, a segunda causa. A análise das tendências recentes mostra que tem havido diminuição lenta mais sustentada das taxas de mortalidade de DAC, no país.

As neoplasias constituem a segunda causa de óbito no país. Entre as mulheres destacam-se o câncer de mama (15\% dos óbitos de câncer), seguido pelo câncer de útero (11\%) e em terceiro lugar, os de traquéia, brônquios e pulmões (9\%); entre os homens destacam-se como principais causas os cânceres de traquéia, brônquios e pulmões (15\%), seguidos pelo câncer de próstata (12\%) e o câncer de estômago (11\%) (MINISTÉRIO DA SAÚDE, 2005). Entre as mulheres a mortalidade por neoplasia de mama apresentou significativo aumento até 1995, com estabilidade das taxas no período seguinte. A partir de 1995 as taxas de mortalidade por câncer do colo do útero apresentaram tendências de crescimento, parcialmente relacionada à melhoria da classificação desta causa.

\section{FATORES DE RISCO RELEVANTES RELACIONADOS À OCORRÊNCIA DAS DCNTS}

Estimativas globais da OMS indicam que um pequeno conjunto de fatores de risco (FR) responde pela grande maioria das DCNTs e por fração substancial da carga de doenças devida a esse grupo de enfermidades. Dentre esses fatores, destacam-se o 
tabagismo, o consumo abusivo de bebidas alcoólicas, a obesidade, o consumo excessivo de gorduras saturadas (que dão origem às dislipidemias), a ingestão insuficiente de frutas e hortaliças e a inatividade física (WHO, 2002).

É possível prevenir a maioria destas doenças, bem como alterar o seu curso, melhorando o prognóstico e qualidade de vida dos indivíduos. Desencadear ações na prevenção dos principais fatores de risco (tabagismo, alimentação inadequada, sedentarismo, hipertensão arterial, obesidade, consumo abusivo de álcool) não somente tem alta efetividade como é custo-efetiva. Como estes fatores de risco são passíveis de modificação, é importante o monitoramento e avaliação destas tendências visando à indução de políticas públicas vis-à-vis a promoção da saúde e a prevenção de doenças. Neste sentido o Ministério da Saúde, em anos recentes, estruturou um sistema de vigilância e monitoramento destes fatores de risco (FR), destacando-se a realização de um inquérito domiciliar em 2003 e a implantação do Sistema de Vigilância de Fatores de Risco e Proteção para Doenças Crônicas não Transmissíveis por Inquérito Telefônico (VIGITEL), em 2006. Com estes instrumentos tornou-se possível acompanhar as tendências de um grupo importante de FRs.

No que se refere à nutrição, em paralelo a intensa redução das prevalências de desnutrição, vem ocorrendo o aumento do sobrepeso e da obesidade. O excesso de peso em adultos aumentou de 18,6\% (1975) para 52,1\% (2010) entre os homens e de $28,6 \%$ para $44,3 \%$ no mesmo período, entre as mulheres. A obesidade passou de $2,2 \%$ (1975) para 14,4\% (2010) entre os homens e de 7,8\% para 15,5\% entre mulheres, no mesmo período (BRASIL, 2011). No centro desta questão está a produção pela indústria de alimentos dos denominados alimentos ultra-processados, que ao conter alto conteúdo calórico são obesogênicos, e necessitam ser intensivamente regulados (MOODIE et al. 2013)

A prevalência do tabagismo no Brasil mostra tendência de declínio. Em 1989, a Pesquisa Nacional de Saúde e Nutrição (PNSN) mostrou que da população de 15 anos ou mais, 33,1\% (40,3\% em homens e $26,2 \%$ em mulheres) fumavam (MS/INAN, 1990). Em 2003, a Pesquisa Mundial de Saúde, mostrou declínio na prevalência para 22,4\% (SZWARCWALD et al., 2005; MONTEIRO et al., 2007) e em 2010, dados do VIGITEL, mostraram prevalência de fumantes no conjunto da população adulta das 27 cidades estudadas de $15,1 \%$, variando de $12,7 \%$ entre as mulheres e $17,9 \%$ entre os homens (BRASIL, 2011). Segundo ainda os dados do VIGITEL, outros fatores de risco para DCNT estão assim distribuídos na população geral: o consumo excessivo de álcool (16,1\%), a inatividade física $(29,2 \%)$, o consumo de carnes com excesso de gordura $(39,2 \%)$, hipertensão (21,6\%) e diabetes (5,3\%). 


\section{5 | CAUSAS EXTERNAS}

As causas externas no Brasil constituem a terceira causa de óbito, com maior proporção de óbitos por agressões e acidentes de transporte terrestre (ATT). Estes óbitos descrevem situação de risco elevado da população jovem, especialmente a masculina, com destaque para os óbitos por armas de fogo. A maioria das vítimas de agressão é do sexo masculino e jovem, com predomínio da faixa etária de 15 a 39 anos. Dos óbitos por agressões, 72\% ocorrem devido a armas de fogo para os homens e 54\% para as mulheres (MINISTÉRIO DA SAÚDE, 2006, REICHENHEIM et al., 2011).

A taxa de mortalidade por ATT na população brasileira em 2007 foi de 23 por 100 mil habitantes, sendo que mais de $80 \%$ das vítimas eram homens e destacaramse os acidentes de trânsito envolvendo pedestres. O risco de mortes por este tipo de acidente entre homens é 4,5 vezes maior do que entre mulheres (MINISTÉRIO DA SAÚDE, 2005; MALTA, et al., 2007c).

Em relação aos homicídios, ocorreram cerca de 48 mil óbitos (28 óbitos por 100 mil habitantes) somente no ano de 2007, numero que é suficiente para expressar a gravidade da situação (REICHENHEIM et al., 2011). A taxa padronizada de mortalidade por arma de fogo apresentou acréscimo entre 1980 e 2003, quase quintuplicando entre homens de 15 a 29 anos (MINISTÉRIO DA SAÚDE, 2005; SOARES FILHO et al., 2007). Em 2004, pela primeira vez em uma década, ocorreram quedas nas taxas de mortalidade por agressão. As mortes relacionadas às armas de fogo declinaram em $8,2 \%$ em relação ao ano anterior, enquanto as hospitalizações relacionadas a este tipo de agressão reverteram tendências históricas e declinaram 4,6 \% em relação a 2003. Este declínio, enquanto lento, tem persistido em anos subseqüentes. Há evidências de que estas mudanças devem-se à legislação mais rigorosa sobre o porte de armas e à campanha de desarmamento entre 2003 e 2004. Estima-se que o impacto destas medidas no primeiro ano da sua implantação foi a redução de 5.563 mortes causadas por armas de fogo (SOUZA et al., 2007b). Existem importantes diferenças no risco de mortalidade segundo raça/cor e condição social (escolaridade). Indivíduos negros (pretos e pardos) associaram-se a pior condição social e ao maior risco de homicídio, comparados aos indivíduos brancos (ARAUJO et al., 2010).

\section{6 | A SITUAÇÃO dE SAÚdE E O DESAFIO DA POBREZA E dA DESIGUALDADE}

A comparação dos indicadores de morbimortalidade apresentados acima com o de outros países de níveis sócio-econômicos semelhantes ajuda a entender melhor e seu significado em termo dos níveis de saúde da população brasileira. Tem sido fartamente documentada a situação paradoxal do Brasil de apresentar indicadores 
econômicos em níveis incompatíveis aos dos indicadores sociais, incluindo-se os de saúde (FLEURY, 1995; OMETTO, 1995). Porem, em anos recentes, possivelmente em resposta a uma serie de medidas no campo social e da saúde, este padrão tem se modificado. Por exemplo, com relação às taxas de mortalidade infantil (TMI) a partir do final da década de 1980 a TMI brasileira inicia tendência de deslocar-se em direção à taxa esperada para um país com o seu nível econômico (PIB percapita similar) (VICTORA et al, 2011). No ano 2000, a TMI para o Brasil era de 28 óbitos por 1000 nascidos vivos enquanto a Argentina, Uruguai e Chile apresentavam TMIs mais baixas, 19, 15 e 9, respectivamente (www.worldbank.org). Em 2008, a TMI para o Brasil era de 17 e para os outros três países de 13, 11 e 7, respectivamente. Isto significou que uma maior redução da TMI foi observada no Brasil, fazendo, como consequência, a sua TMI aproximar-se daquela observada em outros países latino-americanos.

No contexto nacional, a existência de desigualdades inter-regionais pode ser melhor apreendida pelas diferenças entre alguns indicadores de morbimortalidade. No tocante a expectativa de vida ao nascer, enquanto a média nacional em 2007 foi de 73,0 anos, a diferença entre a região Sudeste e Nordeste foi de 4,3 anos (74,4 vs. 70,1 anos) (http://tabnet.datasus.gov.br/cgi/idb2009/a11m.htm). Enquanto para as Regiões Sul e Sudeste as DIs já representavam a quinta causa de óbito em 1980, na região Nordeste, neste mesmo ano, este grupo representava a segunda causa de óbitos e só em anos recentes assume a mesma posição já anteriormente observada para as regiões sul e sudeste.

As diferenças inter-regionais podem ser também visualizadas pelos indicadores de morbidade. Assim, considerando-se a composição de grupos de causas da morbidade hospitalar, dentre os mesmos grupos analisados para a mortalidade, verifica-se que as doenças cardiovasculares representaram a segunda causa de internações nas regiões Sul e Sudeste em 2006, em seguida às doenças respiratórias. Nestas duas regiões, neste mesmo ano, as DIP representaram a quinta causa de internações, respectivamente. Na região Nordeste, as DIP ainda representaram a segunda causa de internações em 2006, enquanto que as doenças cardiovasculares representaram a terceira causa.

A análise histórica de alguns indicadores globais de saúde também evidencia a trajetória do quadro de desigualdades entre as regiões do país. Na década de 30, a região Sudeste apresentava TMI (por 1000 nasc. vivos) de 153, a região Sul 127 e a região Nordeste de 168. Portanto, a região Nordeste apresentava taxas 10\% superiores à região Sudeste e 32\% superiores à Região Sul. No ano de 1997, o Nordeste apresentava TMI de 50,0 sendo esta, no entanto, 280\% e 210\% maiores do que aquelas registradas nas regiões Sul e Sudeste, respectivamente. Somente em anos recentes estas diferenças começam a apresentar sinais lentos de declínio e, em 2007, as diferenças eram de 220\% e 190\%, respectivamente. Em resumo, apesar do fato de que melhorias absolutas foram observadas em todas as regiões, ocorreu a ampliação das diferenças relativas, 
significando uma imensa ampliação das desigualdades regionais (VICTORA et al., 2000), que somente em anos recentes começam a apresentar um pequena inflexão. Outros indicadores de morbidade de base populacional também são reveladores das desigualdades inter-regionais. Por exemplo, as maiores taxas de incidência ou prevalência de muitas doenças infecciosas (p. ex. esquistossomose, doença de Chagas, leishmanioses) nas regiões Norte, Nordeste e Centro-Oeste.

Existem, em todo o mundo, evidências de que a estratificação da população de acordo com seus níveis socioeconômicos define também estratos diferenciais nos níveis de saúde. Países desenvolvidos ou em desenvolvimento, com diferentes patamares nos níveis de saúde das suas populações e com diferentes padrões epidemiológicos, assemelham-se no tocante à existência destes gradientes. A frequência de qualquer doença, com raras exceções, aumenta com a redução do nível social e econômico dos grupos sociais. É consistente o fato de que entre países com níveis econômicos similares, aqueles com maiores níveis de desigualdade social apresentam níveis mais baixos de saúde. Por exemplo, dentre os países desenvolvidos, os Estados Unidos da América, apesar da riqueza, é o que apresenta maiores desigualdades sociais, gerando maiores diferenciais nos indicadores de saúde entre distintos grupos populacionais e, globalmente, é o país com pior nível de saúde neste seleto grupo de países. Organismos internacionais (Banco Mundial, Organização Mundial da Saúde) vêm reconhecendo que sem reduções significativas nas desigualdades sociais será impossível haver melhoras substanciais no quadro global de saúde da população. Consistentemente observa-se que melhorias modestas no padrão das desigualdades têm fortes efeitos nos níveis de saúde. Este aspecto tornou-se tão importante que, em 2005, a Organização Mundial da Saúde criou uma comissão especial para trabalhar com a questão dos determinantes sociais em saúde. Esta produziu um importante relatório sobre o tema que fortalece, internacionalmente, a idéia de intervenções nos determinantes sociais como parte das políticas de saúde (WHO, 2008). Seguindo esta ação da OMS, no Brasil, por ato do Presidente da Republica, foi criada uma comissão similar, a qual apresentou seu relatório também em 2008 (CNDDS, 2008). Em 2011 o Brasil sediou a Conferencia Mundial de Determinantes Sociais em Saúde, convocada pela Organização Mundial da Saúde, e que ratificou a necessidade de ações sobre os determinantes sociais como parte constitutiva das políticas de saúde.

No Brasil, a questão das desigualdades em saúde assume grande importância e ganha nuances especiais dada as imensas desigualdades sociais observadas no país. Por exemplo, em 1999, 50\% dos óbitos infantis concentraram-se nos 30\% dos nascidos vivos que residem nos estados com as maiores taxas de pobreza (DUARTE et al., 2002), bem como a TMI estimada em 1996 entre os 20\% dos brasileiros mais pobres foi 3 vezes maior que a estimada entre os 20\% de maior poder aquisitivo (www.worldbank.org/poverty). A concentração da riqueza e dos bens tem gerado imensas e inaceitáveis distâncias entre os níveis de saúde das populações vivendo em diferentes regiões (como vimos acima), mas também entre aquelas vivendo em 
diferentes espaços das nossas cidades. Assim, no interior das cidades brasileiras quando se comparam as suas zonas mais pobres com as zonas mais ricas são observados imensos diferenciais nos indicadores de saúde. As taxas de mortalidade infantil, por exemplo, podem atingir diferenças de quatro ou cinco vezes entre as áreas mais pobres e as mais ricas de uma mesma cidade. Associam-se a isto imensas desigualdades relacionadas às diferenças de gênero e de grupos étnicos, que agravam ainda mais o quadro geral das desigualdades em saúde. (LEAL et al., 2001; LEITE et al., 2006; MARTINS, 2006; COIMBRA \& BASTA, 2007)

A situação de pobreza e carências em que vive uma parte importante das populações urbanas e rurais, a falta de recursos já reconhecidos como imprescindíveis a bom desenvolvimento físico e mental e as intensas mudanças por que vem passando a nossa sociedade nas últimas décadas como: urbanização acelerada sem adequada infraestrutura urbana (saneamento, habitação, educação), alterações do meio ambiente (como poluição e desmatamentos), ampliação de fronteiras agrícolas, processos migratórios, grandes obras de infraestrutura (rodovias e hidroelétricas), se constituem como a base sobre a qual as doenças dinamicamente ocorrem e sobre o qual o Sistema Único de Saúde (SUS) tem que definir a sua atuação no sentido de atingir não somente a máxima efetividade, mas também ser custo-efetivo, no esforço de maximizar os recursos reconhecidamente limitados. Mostra também a necessidade de medidas estruturais, expressas em políticas públicas em diferentes esferas (econômicas, sociais, ambientais, etc.), que possam impactar sobre as condições de saúde da população. Entende-se que muitos destes problemas não serão equacionados em curto ou médio prazo, no entanto, a superação de alguns deles (p. ex. saneamento, habitação, políticas de alimentação que controlem os níveis de obesidade e a redução das desigualdades sociais) poderão ter forte impacto sobre o quadro global de saúde da população e definir perspectivas de um futuro mais saudável para toda a população brasileira.

\section{OS DESAFIOS COLOCADOS AO SUS}

Vimos acima que as doenças crônico-degenerativas, os acidentes e as diversas formas de violência estão entre as principais causas de óbito e internamentos na população brasileira. Entretanto, observam-se tendências recentes de redução da mortalidade por algumas causas específicas de doenças cardiovasculares, suficiente para gerar redução das taxas para este grupo de causas.

No grupo das doenças infecciosas, além da redução na mortalidade observa-se também diminuição significativa na morbidade. Em outra direção, temos visto o reaparecimento, nas duas décadas passadas, de problemas como a cólera e a dengue, que além de exporem as deficiências ambientais urbanas em nosso país, tornam parcelas importantes da população vulneráveis a doenças que deveriam estar 
superadas, amplificando a já alta carga de doenças da população. Alem disto, se tornam fonte de tensão social, dado ao potencial epidêmico de doenças, como a dengue. A isto, agrega-se a possibilidade de novas formas de manifestação das doenças na população (p. ex. o aumento na severidade por surgimento de novas cepas patogênicas, ou a ampliação da resistência aos medicamentos antimicrobianos), bem como a persistência de problemas como a tuberculose e a hanseníase, que se esperavam estar superados. Como conseqüência, mesmo para o grupo das DIs faz-se necessário manter estruturas de altos custos para vigilância e atenção à saúde, recursos que, em caso dos problemas anteriores estarem superados, poderiam vir a ser utilizados em problemas de saúde com maior magnitude e para os quais existem menores possibilidades de prevenção em curto prazo.

Um quadro bem paradigmático desta superposição de padrões de doenças e problemas de saúde ocorre com relação aos problemas nutricionais. Reduções na prevalência da desnutrição estão acontecendo em paralelo ao crescimento da prevalência do excesso de peso e da obesidade. Deve-se destacar também que, em geral, o tratamento das "doenças da modernidade" requer mais recursos tecnológicos e, como conseqüência, implica em maiores custos para o sistema de saúde. A obesidade, com seu efeito potencializador sobre a ocorrência de uma série de DCNTs, caracteriza bem esta situação. Na mesma direção, a violência que, ao lado das mortes e incapacidades na população produtiva, implica em pesadas sobrecargas sobre o sistema de saúde e o sistema previdenciário. Estes fatos destacam a importância de investimentos em ações de promoção à saúde e prevenção de doenças que sejam custo efetivas e tenham impacto na redução dos custos econômicos e sociais dos agravos à saúde e na melhoria da qualidade de vida (BARRETO \& CARMO, 1998; MALTA, et al., 2007a).

A falta de solução para alguns problemas estruturais básicos (p.ex. o saneamento), a manutenção de condições e modos de vida inadequados e altos padrões de desigualdades, a insuficiência dos mecanismos que regulam os danos ao meio ambiente, a indústria de alimentos, entre outros importantes e modernos determinantes do estado de saúde da população, ocasionam que os riscos aos quais está exposta a população se superponham, amplificando os seus efeitos negativos sobre a saúde. Assim, se de um lado temos a manutenção dos problemas urbanos, caracterizados por marcantes deficiências em áreas como o saneamento ambiental, habitação e transporte, temos também o aumento da poluição ambiental de origem química (industrial, inseticidas, etc.), o desmatamento e outras formas de agressão ambiental que afetam o campo e as cidades, os riscos ocupacionais, o crescimento dos fatores psicossociais estressores, as mudanças comportamentais e de hábitos alimentares e as desigualdades intraurbanas. Ademais, o fato do envelhecimento da população ocorrer em condições em que se associam múltiplos riscos amplifica as chances de aumento da carga de morbidade na população idosa, aumentando a taxa de adoecimento e reduzindo a qualidade de vida deste grupo populacional. 
Existem cada vez mais relatos sobre os efeitos deletérios de fatores que ocorrem no plano internacional sobre a saúde. Os efeitos da globalização que se relaciona com questões tão aparentemente diversas como o aumento das desigualdades entre as nações, a intensificação do comércio internacional, em especial o comércio de produtos alimentícios, o narcotráfico e o contrabando de armas e cigarros, ou o aumento da temperatura global ocasionada pela crescente "efeito estufa" gerado pela poluição atmosférica, são exemplos de "novos riscos globais" com efeitos na deterioração das condições de saúde 'local'. Este complexo processo de superposição de fatores econômicos, sociais e ambientais, velhos ou novos, globais ou locais, cria as condições para que algumas doenças infecciosas tradicionais sejam mantidas e que ocorra a emergência ou a reemergência de outras tantas, mas também estão entre os determinantes do aumento das doenças crônicas e das violências.

Tendo em vista a impossibilidade de ações a curto ou mesmo em médio prazo na minimização de alguns importantes determinantes da saúde gerados por processos sejam locais ou globais, faz-se urgente a superação de alguns determinantes tradicionalmente vinculados à ocorrência de doenças e cuja superação pode ser alcançada em função de decisões políticas e recursos mais limitados. Por exemplo, a universalização do acesso à água potável, que não está muito longe de ser atingido, e do esgotamento sanitário, para o qual maiores investimentos se fazem necessários, bem como a universalização do acesso a atenção básica de saúde de qualidade e os programas de transferência condicional de renda (em especial o Bolsa Família) podem ter impactos significativos em dimensões importantes das condições de saúde da população brasileira.

Avançar em políticas custo-efetivas de promoção da saúde e prevenção das doenças é fundamental para a melhoria da qualidade de vida das populações, intervindo em importantes determinantes do processo saúde doença, ampliando a consciência publica sobre a magnitude dos seus problemas e como contribuir para superá-los, além de ampliar o diálogo e estimular a integração das ações na direção da produção de políticas integradas e com potencial salutogênico. Consideremos, por exemplo, um fator de risco das DCNTs - o sedentarismo. Para a sua redução, além de informações qualificadas à população quanto aos benefícios da prática de atividade física, são necessárias ações intersetoriais que ampliem os espaços públicos acessíveis e seguros para a prática de atividades físicas e do lazer. Para se tornarem sustentáveis estas políticas teriam que se desdobrar em debates e ações sobre os processos de urbanização e de organização da mobilidade urbana de modo a estimular o envolvimento da população na busca de estilos de vida fisicamente mais ativos. Da mesma forma, a garantia da alimentação saudável implica entre outras, em ações voltadas para ampliação da produção e do acesso a alimentos saudáveis, forte regulamentação da indústria alimentar em relação ao uso de sal, gorduras saturadas, conteúdo calórico, dentre outras. (BRASIL, 2008). 
A superposição de problemas de saúde implica na manutenção de alta carga de morbidade e mortalidade sobre cada individuo e sobre a população. A redução desta carga representa reduzir a morbidade global que aflige os indivíduos no curso da vida, comprimindo a morbidade individual e a carga mórbida das populações. Benefícios alcançados no curso das ultimas décadas necessitam ser ampliados para outras causas de adoecimento e morte da população. Os resultados positivos indicam que mesmo para doenças para as quais existem tecnologias de saúde efetivas, os efeitos são muito mais intensos e duradouros em contextos favoráveis em que as ações de saúde integradas às demais políticas econômicas, sociais e ambientais. Para realçar esta questão, lembramos que as situações em que as melhorias dos níveis de saúde não são alcançados de forma homogênea por todos os grupos sociais, em especial os grupos com maior carga de doenças (não por acaso, em geral os grupos mais pobres) os níveis globais de saúde permanecem baixos ou crescem em velocidade aquém do esperado. Portanto, a equidade em saúde não pode se resumir a equidade de acesso ao sistema de saúde, tem que ser pensada integrada a políticas econômicas, sociais e ambientais também equânimes, que permitam a redução das inequidades sociais, em geral. Desta forma, reduzindo a carga mórbida que tais determinantes exercem na ocorrência de doenças e sofrimentos, em especial sobre as classes mais baixa da sociedade.

Tudo isto não retira o importante papel dos sistemas de saúde, seja na cura (tratamento) ou na prevenção (p. ex. vacinas, assistência pré-natal) das doenças. Por exemplo, evidências recentes do Brasil têm mostrado que a Estratégia de Saúde de Família, a principal abordagem para a atenção básica no SUS, além da expansão das ações de saúde vem tendo impacto sobre os níveis de saúde ao contribuir para a redução significativa das taxas de mortalidade infantil, pós-neonatal e neonatal (AQUINO et al., 2009; MACINKO et al., 2006; MACINKO et al., 2007), mortalidade por diarréia e infecções respiratórias (RASELA et al., 2010) e internamentos por causas sensíveis a atenção primaria a saúde (DOURADO et al., 2011). Estes achados sinalizam a importância da atenção primária como elemento fundamental do sistema de saúde e como importante elo com um conjunto de políticas e ações que interferem em determinantes da saúde da população, por exemplo, o programa Bolsa Família ao transferir renda para as famílias muito pobres, mas ao atrelar isto a condicionalidades que incluem ações no sistema de saúde. Para atender as condicionalidades de saúde, crianças menores de 7 anos devem ser vacinadas de acordo com o calendário brasileiro de imunização e devem ser rotineiramente acompanhadas para o registro do crescimento e desenvolvimento. Mulheres grávidas e lactantes devem comparecer a consultas pré-natal e pós-natal programadas e atividades educacionais de saúde e nutricionais. Se possível, as condicionalidades relacionadas com a saúde devem ser cumpridas utilizando as instalações do principal programa de cuidados primários a saúde do país, nomeadamente o Programa de Saúde da Família (PSF). Como resultado, existem evidências de que a ação do programa Bolsa Familia não somente contribui para 
redução da desnutrição (PAES-SOUZA et al., 2011) como das taxas de internamentos hospitalares e de mortalidade de criancas (RASELLA et al., 2013).

\section{8 | FUNDAMENTOS PARA TRAÇAR POSSÍVEIS CENÁRIOS DA SITUAÇÃO DE SAÚDE EM 2022-2030}

Mesmo partindo-se do quadro apresentado das tendências recentes das doenças e outros problemas de saúde que afligem a população brasileira, e do intrincado conjunto de determinantes envolvidos, os esforços para traçar o quadro da situação de saúde da população brasileira para o período de 2022-30 envolve grandes incertezas. Este estará em grande parte na dependência da implementação de uma série de políticas e outras ações publicas potencialmente efetivas e que venham a impactar em um conjunto complexo de determinantes. Depende ainda da consolidação da rede de atenção básica a saúde que tenha capacidade resolutiva e seja de qualidade, e ainda do desenvolvimento cientifico que dê suporte as ações planejadas e que estimule o surgimento de inovações que tragam soluções para problemas atualmente existentes e para os quais faltam conhecimentos ou tecnologias adequadas.

Os diversos grupos de doenças responderão de forma diferente às diferentes políticas e ações. Assim, enquanto algumas ações têm efeitos em uma gama larga de problemas, outras terão efeitos em questões focais e mais específicas. Por exemplo, a redução dos atuais níveis de desigualdade, seja social ou regional, pode ter efeitos em uma gama diversificada de problemas de saúde; ampliação do acesso ao saneamento terá efeitos em um grupo de doenças infecciosas; o desenvolvimento de novas vacinas interferirá na ocorrência de doenças especificas; enquanto um novo tratamento farmacológico modifica o curso de uma doença já instalada. Evidentemente, devemos ter em conta que as modificações dos padrões de morbimortalidade estarão na dependência da interação e do equilíbrio de um complexo de diferentes ações. Ter o sistema de saúde como único vetor de modificação da situação de saúde não encontra respaldo nas evidências cientificas existentes. Pelo contrário, existem evidências de que as grandes modificações nos níveis de saúde, ocorridas em alguns momentos da história, aconteceram por ações externas ao sistema de saúde. No entanto, em épocas mais recentes, desenvolvimentos científicos e tecnológicos no campo da saúde têm sido importantes quer seja para modificar o curso da ocorrência de algumas doenças, destacando-se aqui o papel das vacinas, quer impactando no curso de doenças já instaladas, através de tratamentos farmacológicos (destaque aos antibióticos e antihipertensivos entre outros) e não farmacológicos (como as cirurgias).

Portanto, o sistema de saúde, da forma que é concebido, tem um papel central e importante na cura ou amenização das doenças e um papel menor na 
modificação dos padrões de morbidade ou mortalidade prevalentes em uma dada sociedade. Os determinantes econômicos, sociais, ambientais e comportamentais têm papel dominante na definição destes padrões. Isto pode ser exemplificado pelo fato de que intensas mudanças nos padrões de saúde dos Europeus e Norte-Americanos aconteceram entre o final do Século XIX e início do século XX, momento em que as tecnologias de saúde eram ainda rudimentares e de baixa efetividade. Por outro lado, em épocas recentes, temos o exemplo dos EUA, que após sua população ter atingido níveis recordes de saúde apresenta, em anos recentes, claros sinais de retrocesso, em decorrência da manutenção e agravamento das desigualdades sociais e de mudanças nos estilos de vida verificados em décadas recentes (MICHAUD et al., 2011).

\section{9 | DOENÇAS INFECCIOSAS (DIS) EM 2022}

Até meados da década de 1970 acreditava-se que as DIs iriam paulatinamente desaparecer da face da terra, primeiramente nos países desenvolvidos e em seguida e de forma mais gradual nos países pobres e em desenvolvimento. Porem, esta crença foi abalada pelo aparecimento de novas doenças infecciosas (p.ex. AIDS) ou pelo ressurgimento de doenças que se encontravam em fase avançada de controle (p. ex. a dengue). Hoje, existe consenso de que se por um lado uma parte destas doenças pode ser controlada e, eventualmente, algumas erradicadas (p.ex. a varíola), algumas outras, enquanto possíveis de virem a ser controladas, ainda apresentam incertezas sobre quando isto ocorrerá (p.ex. malária, hanseníase, tuberculose), outras não apresentam sinais de que estarão sob controle em um prazo razoável (p.ex. dengue). Adiciona-se a isto possibilidade de algumas delas conhecidas ou ainda não conhecidas se apresentem na forma de epidemias (ou mesmo pandemias) criando emergências de saúde pública, com imenso impacto na saúde, na economia e no cotidiano das populações atingidas.

O avanço no controle das DIs, composto por um conjunto diversificado de doenças e seus agentes infecciosos e que respondem a diferentes tipos de determinantes, está na dependência da ampliação e consolidação de políticas que modificam tais determinantes e que potencializem as ações de saúde voltadas para cura e prevenção, o que inclui, em vários casos, avanços científicos e tecnológicos necessários. Por exemplo, faz-se urgente o desenvolvimento de vacinas para algumas doenças (p.ex. dengue) ou novos tratamentos (p. ex. leishmaniose visceral).

No tocante as DIs, em geral, pode-se delinear para 2022/2030 um quadro razoavelmente positivo tendo em vista os avanços até então observados na implementação de uma série de políticas que afetam determinantes de muitas destas doenças, incluindo a atenção primária e a vigilância à saúde. Entretanto, para 
chegarmos a um estágio ainda mais avançado de controle do conjunto das DIs em 2022, principalmente daquelas doenças que contribuem com alta carga de morbidade ou que têm potencial epidêmico, faz-se necessário:

1. Avanço nas políticas de fornecimento de água potável e principalmente de saneamento básico, crucial para a consolidação e para dar sustentabilidade ao controle de uma gama diversa de problemas que atinge, em especial, a população de crianças. Estes problemas incluem as diarréias causadas por agentes de transmissão oral-fecal, as helmintoses intestinais, a esquistossomose, a hepatite A, a leptospirose entre outras. Caso em 2022 tenha-se ampliado a cobertura de acesso a água potável em áreas rurais e de saneamento básico em áreas urbanas e rurais, ter-se-ão dadas as condições básicas para colocar este grupo de doenças em estagio avançado de controle.

2. Melhorias no ambiente urbano (saneamento, melhorias habitacionais, melhoria geral da higiene, etc.) podem trazer importante impacto nas doenças de transmissão fecal-oral, mas também de outras DIs importantes no contexto urbano sejam de transmissão através do contato interpessoal (p.ex., tuberculose, hanseníase) ou por outras vias (p. ex. leptospirose).

3. O fortalecimento da atenção primaria à saúde, ampliando e consolidando o acesso amplo para todas as faixas populacionais e regiões, poderá, através do tratamento precoce e efetivo, ter impacto no curso de uma serie de enfermidades (p. ex. diarréias, infecções respiratórias, tuberculose, hanseníase, malária);

4. O desenvolvimento de inovações biotecnológicas, como vacinas e tratamentos, poderá ser central em acelerar o controle de algumas doenças (p. ex. dengue, leishmaniose visceral) ou consolidar o controle de algumas outras (p.ex. AIDS, malária, tuberculose, hanseníase).

5. Um problema a ser considerado são as infecções nosocomiais, cuja magnitude ainda não está bem estabelecida no país. Apesar de não termos clareza sobre suas tendências, pela experiência internacional estas tendem a crescer em paralelo ao crescimento dos recursos tecnológicos em saúde e que incluem o aumento na frequência das hospitalizações, o prolongamento da vida, as técnicas invasivas para diagnostico e tratamento, o uso de medicações imunossupressoras, entre outras. 


\section{0 | DOENÇAS CRÔNICAS DEGENERATIVAS EM 2022}

Apesar de tendência observada de redução das taxas de mortalidade de algumas doenças deste grupo, graças ao desenvolvimento de tecnologias médicas e do acesso de uma maior parte da população às mesmas, existem evidências e claras sinalizações de que outros problemas da área das DCNTs e alguns dos seus fatores de risco apresentam tendências crescentes. A não solução de alguns destes problemas-chave poderão causar um cadeia de eventos que levará a efeitos danosos na saúde da população e uma crescente demanda aos serviços de saúde. Os principais problemas são:

1. O aumento da prevalência da obesidade em adultos, mas também em crianças, tem sido observado em todo o mundo, não sendo diferente no Brasil. Uma das implicações mórbidas mais imediatas da obesidade é o diabetes, havendo evidências de que a prevalência da diabetes dobrará até 2030 nas Américas e o Brasil, certamente, seguirá esta tendência (http://da3.diabetesatlas.org/ downloadables/Graphics/index.html). A projeção da tendência observada em anos recentes de aumento da prevalência de obesidade faz prever que em pouco mais de uma década atingiremos níveis próximos a atual situação nos EUA (SCMIDT et. al., 2011). Portanto, o termo "epidemia de obesidade" reflete bem a atual situação em que vivemos. Enquanto não haja consenso sobre as medidas mais efetivas para a contenção desta epidemia, estas se centram em políticas e ações de promoção de dietas saudáveis e de estilos de vida ativo, em todas as etapas do curso da vida. Esforços por uma dieta saudável incluem ações em diferentes níveis, como o estímulo ao aleitamento materno, dietas saudáveis na escola e na alimentação do trabalhador, enérgica regulação da indústria alimentar que desestimule os alimentos hipercalóricos e com alto teor de sódio e de gorduras saturadas, políticas fiscais e agrícolas que aumentem a produção e o acesso da população a alimentos saudáveis como frutas e legumes e desestimulem os alimentos hiperprocessados. Intervenções na estrutura urbana que estimulem o hábito de atividades físicas regulares, as caminhadas, usos de bicicletas etc. são medidas complementares importantes.

2. Conectadas com a situação apresentada no item anterior, temos as doenças cardiovasculares, que apesar de apresentarem mortalidade com tendência declinante, por varias décadas continuam como a primeira causa de óbito e de hospitalizações no país. Este grupo inclui o infarto do miocárdio e os acidentes 
cerebrovasculares que podem ter efeitos devastadores e acontecer precocemente. Políticas que estimulem ações de contenção aos potencias fatores de risco incluem dietas saudáveis, normocalóricas, com baixo conteúdo de sódio e gorduras e estímulo a atividade física. Como o tratamento da hipertensão arterial é um fator importante para quebrar a cadeia de causas das DCVs, a ampliação de acesso aos serviços básicos de saúde, bem como acesso ao tratamento é um reforço importante aos programas de controle, através do seu diagnostico e tratamento precoce. A recente medida do Ministério da Saúde, dando amplo acesso gratuito a medicamentos para o tratamento da hipertensão, pode vir a ter impacto na redução deste grupo de doenças.

3. Os diferentes tipos de câncer vêm apresentando tendências diferentes de mortalidade, algumas de redução e outras de aumento. Em homens se destacam o aumento das taxas de mortalidade do câncer de próstata e a redução do câncer de estomago; entre mulheres destacam-se os aumentos em câncer de mama e de pulmão. No tocante a ocorrência dos cânceres de mama e cervical, suas taxas de incidência entre as brasileiras estão entre as mais altas do mundo. Assim com ocorre com o câncer do esôfago, principalmente entre os homens. Além disto, as taxas de sobrevivência estão abaixo daquelas observadas em países desenvolvidos, refletindo diagnóstico tardio e falhas nos tratamentos. Para alcançarmos um quadro de saúde com redução da ocorrência dos cânceres em geral, mas especialmente daqueles grupos para o qual existem fatores de risco bem estabelecido ou para os quais existem tratamentos efetivos, algumas medidas deverão ser implementadas. As medidas de redução do habito de fumar e da ingestão do álcool podem ter efeito na redução de alguns tipos de câncer (pulmão, laringe, esôfago, etc.). Dietas saudáveis e atividades físicas regulares podem ter impacto importante na ocorrência de diversos cânceres. Estima-se que em torno de um quinto dos cânceres estejam associados a infecções. Entre estes incluem as hepatites B e C, associadas a câncer de fígado, e o papilomavirus, associado ao câncer cervical. Para a hepatite B já existe uma vacina em uso e adotada no esquema nacional de vacinação. Para o papilomavirus também já existe uma vacina disponível, porem como não há completo acordo sobre a sua efetividade, no Brasil, não foi incluída entre as vacinas do programa nacional de vacinação. $O$ fortalecimento do sistema de saúde, permitindo o diagnóstico precoce e o acesso 
a tratamento adequado será também um importante fator para termos redução deste conjunto de patologias, em especial para os tipos que dispõem de tratamentos efetivos.

4. As doenças respiratórias crônicas, em especial a doença pulmonar obstrutiva crônica (DPOC) e asma dão importantes contribuições à carga global de mortalidade e de morbidade. Entretanto, as tendências destes indicadores são decrescentes, o que torna alvissareira a perspectiva para a próxima década. A redução observada nas hospitalizações e mortes por DPOC deve ter como sua principal causa as reduções observadas no hábito de fumar nas últimas décadas ou outras melhorias na qualidade do ar respirado. Reduções subsequentes no hábito de fumar e políticas de controle da poluição urbana podem trazer maiores reduções na sua ocorrência. Quanto à asma não há indícios de que venha ocorrendo reduções significativas na sua ocorrência, e pouco se conhece dos seus fatores de risco, assim a redução observada na mortalidade e hospitalizações tem sido atribuída ao maior acesso a tratamentos efetivos. Portanto, uma combinação de medidas de prevenção efetivas, no caso da DPOC, e acesso a serviços de saúde e adequado tratamento podem ser cruciais para termos mais baixas taxas de morbidade e mortalidade deste grupo de patologias em 2022.

5. No tocante às doenças neuropsiquiátricas, enquanto não tenham maior impacto na mortalidade, as evidências existentes mostram a sua alta prevalência na população e os seus efeitos sobre o cotidiano dos indivíduos acometidos, das suas respectivas famílias e de todos aqueles envolvidos em suas relações sociais. Este grupo de doenças, por sua vez, constitui fatores de risco para outras doenças infecciosas e crônicas. Indivíduos com distúrbios neuropsiquiátricos têm frequentemente reduzida a capacidade de auto-cuidar-se, tornando-se mais susceptíveis a uma gama variada de doenças. Em geral, não existem dados suficientes que permitam inferir sobre as tendências no tempo deste grupo de doenças. No entanto, as demências parecem estar aumentando seguindo o processo de envelhecimento populacional. A taxa de mortalidade associada às demências aumentou de aproximadamente quatro vezes no período 1996 a 2007 (1,8 para 7,0/100.000 hab.). A falta de conhecimentos precisos sobre os complexos determinantes destas doenças tornam mais difíceis as previsões sobre o seu curso em populações. Entretanto, espera- 
se que melhorias gerais nas condições de vida do brasileiro, associadas com reduções nas desigualdades sociais possam ter papel redutor na sua ocorrência.

\section{1 | VIOLÊNCIA E LESÕES EM 2022}

Em anos recentes, um conjunto de políticas tem sido implementadas no país no esforço de conter as diversas formas de violência e lesões que, nas últimas décadas, colocaram este grupo de problemas de saúde entre os mais expressivos que afligem a população brasileira. Estas políticas cobrem um conjunto diversificado de intervenções no esforço de reduzir em especial os dois principais componentes em termos do impacto na morbidade e na mortalidade: os homicídios e os acidentes de trânsito. Enquanto suas taxas de mortalidade continuem abusivamente altas, as evidências é de que ambas se encontram em lento descenso no país. Não sendo este, entretanto, um processo uniforme em todas as regiões do país e para os seus diferentes subtipos. Por exemplo, os homicídios, enquanto apresentem sinais de descenso na região Sudeste, com redução de mortalidade de 15\% entre 1991 e 2007, na região Nordeste houve aumento de 19,5\% no mesmo período. Com relação aos acidentes de trânsito enquanto haja uma tendência, também lenta, de queda global da mortalidade, os óbitos associados a acidentes envolvendo motocicletas crescem de forma vertiginosa. Enquanto em 1996 acidentes envolvendo motocicletas totalizavam $4 \%$ dos óbitos por acidentes de trânsito, em 2007 já representavam 28\%. Portanto, o quadro em 2022 dependerá da continuidade de medidas que já estão em curso e outras que venham a ser implementadas com vistas a reduzir os homicídios e outras formas de acidentes. Em relação às mortes por motocicletas medidas urgentes precisam ser implementadas para reverter a atual tendência. Nessa área, o fosso que separa o Brasil dos países desenvolvidos ou mesmo de outros países em desenvolvimento é muito grande. A taxa nacional de mortalidade por homicídios em 2007 era vinte vezes maior que a China e 5,5 vezes maior que a Argentina, sendo ligeiramente menor apenas do que as observadas na Colômbia e na África do Sul. As tendências recentes não mostram, em geral, importantes inflexões para modificar as diferenças apresentadas, fazendose necessárias ações mais vigorosas que associem medidas especificas (segurança, melhoria das vias publicas, etc...) com amenizações de macrodeterminantes, como as desigualdades sociais. 


\section{2 | REFERÊNCIAS BIBLIOGRÁFICAS}

ALMEIDA-FILHO, N, et al. Brazilian multicentric study of psychiatric morbidity. Methodological features and prevalence estimates. Br J Psychiatry. 171:524-9, 1997.

AQUINO R, DE OLIVEIRA NF, BARRETO ML. Impact of the family health program on infant mortality in Brazilian municipalities. Am J Public Health. Jan;99(1):87-93, 2009.

AQUINO R, BARRETO ML. Programa de Saúde da Família: acerca da adequação do uso do seu indicador de cobertura. Cad Saúde Publ, Rio de Janeiro, 24(4):905-914, abr, 2008.

ARAUJO, EDNA MARIA DE et al. Spatial distribution of mortality by homicide and social inequalities according to race/skin color in an intra-urban Brazilian space. Rev. bras. epidemiol. [online]. 2010, vol.13, n.4 pp. 549-560. Disponível em <http://www.scielo.br/scielo.php?script=sci_ arttext\&pid=S1415-790X2010000400001\&lng=en\&nrm=iso>.

BARATA RB, DE ALMEIDA MF, MONTERO CV, DA SILVA ZP. Health inequalities based on ethnicity in individuals aged 15 to 64, Brazil, 1998. Cad Saude Publica. 23 (2):305-13, 2007.

BARRETO ML, CARMO EH. Situação da saúde da população brasileira: tendências históricas, determinantes e implicações para as políticas de saúde. Informe Epidemiológico do SUS, ano III (nos 3 / 4): 5-34, 1994.

BARRETO ML, CARMO EH. Tendências recentes das doenças crônicas no Brasil. In: LESSA I (org.). $\mathrm{O}$ adulto brasileiro e as doenças da modernidade: epidemiologia das doenças crônicas não transmissíveis. São Paulo: Hucitec, Rio de Janeiro: Abrasco, 1998. p. 15-27.

BARRETO, M. L.; CARMO, E. H. Determinante das condições de saúde e problemas prioritários no país. In: BRASIL. MINISTÉRIO DA SAÚDE. Caderno da $11^{\text {a }}$ Conferência Nacional de Saúde. Brasília: Ministério da Saúde, 2000. p. 235-259.

BARRETO ML, CARMO EH, SANTOS CAST, FERREIRA LDA. Saúde da população brasileira: mudanças, superposição de padrões e desigualdades. In: FLEURY S (Org.). Saúde e Democracia - A Luta do CEBES. São Paulo: Lemos Editora, 1997, pp.45-60.

BARRETO, M.L.; TEIXEIRA, M.G. \& CARMO, E.H.,. Infectious diseases epidemiology (Glossary). Journal of Epidemiology and Community Health, 60:192-195, 2006.BARRETO ML, et al. Effect of city-wide sanitatiion programme on reduction in rate of childhood diarrhoea in northeast Brazil: assessment by to cohort studies. Lancet, 370 (9599): 1622-8, 2007.

BARRETO ML, et al. Successes and failures in the control of infectious diseases in Brazil: social and environmental context, policies, interventions, and research needs. Lancet, 377 (9780):187789, 2011.

BRASIL. MINISTÉRIO DA SAÚDE. VIGITEL Brasil. Vigilância de fatores de risco e proteção para doenças crônicas por inquérito telefônico: estimativas sobre frequência e distribuição sóciodemográfica de fatores de risco e proteção para doenças crônicas nas capitais dos 26 estados brasileiros e no Distrito Federal em 2006. Brasília: Ministério da Saúde; 2007.

BRASIL. MINISTÉRIO DA SAÚDE. Saúde Brasil 2004 - uma análise da situação de saúde. Brasília: Ministério da Saúde, 2004. 
BRASIL. MINISTÉRIO DA SAÚDE. Saúde Brasil 2005: uma análise da situação de saúde no Brasil. Brasília: Ministério da Saúde, 2005.

BRASIL. MINISTÉRIO DA SAÚDE. Saúde Brasil 2006: uma análise da desigualdade em saúde. Brasília: Ministério da Saúde, 2006.

BRASIL. MINISTÉRIO DA SAÚDE. Saúde Brasil 2006: uma análise da situação de saúde no Brasil. Brasília: Ministério da Saúde; 2006. 620 p.

BRASIL. MINISTÉRIO DA SAÚDE Diretrizes e recomendações cuidado integral de doenças crônicas não-transmissíveis. Promoção da saúde, vigilância, prevenção e assistência. Ministério da Saúde. Brasília, DF, 2008. No prelo.

BRASIL. PRESIDÊNCIA DA REPÚBLICA. Decreto de 13 de março de 2006: cria a Comissão de Determinantes Sociais em Saúde. Brasília, DF, 2006.

CARMO E H, BARRETO ML, SILVA-JR, J B. Mudanças nos padrões de morbimortalidade da população brasileira: os desafios para um novo século. Epidemiologia e Serviços de Saúde, 1: 63-76, 2003.

COIMBRA CE JR, BASTA PC. The burden of tuberculosis in indigenous peoples in Amazonia, Brazil. Trans R Soc Trop Med Hyg. 101(7):635-6, 2007.

DOURADO I, et al. Trends in primary health care-sensitive conditions in Brazil: the role of the Family Health Program (Project ICSAP-Brazil). Med Care. Jun;49(6):577-84, 2011.

DUARTE EC, et al. Epidemiologia das Desigualdades em Saúde no Brasil : um estudo exploratório. OPAS, Brasília, 2002.

FLEURY S. Iniquidades nas políticas de saúde pública: o caso da América Latina. Rev Saude Publica, 29(3)243-50, 1995.

GUERRA MR, GALLO CVM, MENDONÇA GAS. Risco de câncer no Brasil: tendências e estudos epidemiológicos mais recentes. Rev Bras Cancerol 51(3): 227-234, 2005.

ISAAC steering committee. Worldwide variation in prevalence of symptoms of asthma, allergic rhinoconjunctivitis, and atopic eczema: ISAAC. Lancet, 351:1225-1232, 1998.

LAURENTI R. O perfil da mortalidade materna. In: MINAYO MCS (Org.). Os muitos Brasis: saúde e população na década de 80. São Paulo - Rio de Janeiro, HUCITEC- ABRASCO, 1995.

LAURENTI R, MELLO JORGE MHP, GOTLIEB SLD. A mortalidade materna nas capitais brasileiras: algumas características e estimativa de um fator de ajuste Rev. Bras. Epidemiol. 7(4):449-60 2004

LEAL M DO C, DA GAMA SG, DA CUNHA CB. Desigualdades raciais, sociodemográ-ficas e na assistência ao pré-natal e ao parto, 1999-2001. Rev Saude Publica. 39 (1):100-7, 2005.

Leite MS, et al. Crescimento físico e perfil nutricional da população indígena Xavánte de Sangradouro-Volta Grande, Mato Grosso, Brasil. Cad Saude Publica. 22(2):265-76, 2006.

MACINKO J, DE SOUZA MFM, GUANAIS FC, DA SILVA SIMOES CC. Going to scale with communitybased primary care: An analysis of the family health program and infant mortality in Brazil, 1999-2004. Soc Sci Med. 65(10):2070-80, 2007. 
MACINKO J, GUANAIS FC, DE FATIMA M, DE SOUZA M. Evaluation of the impact of the Family Health Program on infant mortality in Brazil, 1990-2002. J Epidemiol Community Health. 60(1):13-9, 2006.

MALTA DC, et al. Construção da vigilância e prevenção das doenças crônicas não trasmissíveis no contexto do sistema único de saúde. Epidemiologia e Serviços de Saúde, 15: 47-64, 2006.

MALTA DC, et al. Tendência de mortalidade do Câncer de pulmão, traqueia e brônquios no Brasil - 1980-2003. Jornal Brasileiro de Pneumologia, 2007 b.

MALTA DC, et al. Análise descritiva e de tendência de acidentes de transporte terrestre para políticas sociais no Brasil. Epidemiologia e Serviços de Saúde, Brasília, 2007c.

MARTINS AL. Mortalidade materna de mulheres negras no Brasil. Cad Saude Publica. 22(11):2473-9, 2006.

Michaud PC, et al. Differences in health between Americans and Western Europeans: Effects on longevity and public finance. Soc Sci Med. Jul;73(2):254-63, 2011.

MONTEIRO CA, et al. The nutrition transition in Brazil. Eur J Clin Nutr, 49(2):105-13, 1995.

MONTEIRO CA, et al. Population-based evidence of a strong decline in the prevalence of smokers in Brazil (1989-2003). Bull World Health Organization, 85:527-34, 2007.

MONTEIRO CA, et al. Causes for the decline in child under-nutrition in Brazil, 1996-2007. Rev Saude Pública. 43(1):35-43, 2009.

MOODIE R,et al. LANCET NCD ACTION GROUP. Profits and pandemics: prevention of harmful effects of tobacco, alcohol, and ultra-processed food and drink industries. Lancet, Feb 23;381(9867):670-9, 2013.

MOURA EC, et al. Vigilância de Fatores de Risco para Doenças Crônicas por Inquérito Telefônico nas capitais dos 26 estados brasileiros e no Distrito Federal (2006). Revista Brasileira de Epidemilogia. 11(supl 1): 20-37, 2008.

NUNES M, JUCÁ VJV, BRANCA CP. Ações de saúde mental no Programa Saúde da Família: confluências e dissonâncias das práticas com os princípios das reformas psiquiátrica e sanitária. Cad. Saúde Pública, 23(10):2375-2384, 2007.

OMETTO AMH, FURTUOSO MCO, SILVA MV. Economia brasileira na década de oitenta e seus reflexos nas condições de vida da população. Rev Saude Pública, 29(5):403-15, 1995.

PAES-SOUSA R, SANTOS LM, MIAZAKI ÉS. Effects of a conditional cash transfer programme on child nutrition in Brazil. Bull World Health Organ, 89(7):496-503, 2011.

PAIM JS,COSTA MC.Decrease and inequalities of infant mortality in Salvador, 1980-1988]. Bol Oficina Sanit Panam, May;114(5):414-28, 1993.

RASELLA D, AQUINO R, BARRETO ML. Reducing Childhood Mortality From Diarrhea and Lower Respiratory Tract Infections in Brazil. Pediatrics, 126(3): e534-e540, 2010.

RASELLA D, et al. The impact of a conditional cash transfer programme on childhood mortality in Brazil, Submetido para publicação, 2013. 
REICHENHEIM ME, et al. Violence and injuries in Brazil: the effect, progress made, and challenges ahead. Lancet, Jun 4;377(9781):1962-75, 2011.

RONCALLI AG. Levantamentos epidemiológicos em saúde bucal no Brasil. In: Peres MA, Antunes JLF, editores. Epidemiologia em saúde bucal. Rio de Janeiro: Guanabara Koogan, 2006.

SCHMIDT MI, et al. Chronic non-communicable diseases in Brazil: burden and current challenges. Lancet, Jun 4;377(9781):1949-61, 2011.

SOARES FILHO AM, et al. Análise da mortalidade por homicídios no Brasil. Epidemiologia e Serviços de Saúde, Brasília, 2007.

SOUZA MFM, et al. Reductions in Firearm-related Mortality and Hospitalizations in Brazil Following the Introduction of National Gun Control Measures. Health Aff (Millwood), 26(2):57584, 2007.

SOUZA-MACHADO C, et al. Rapid reduction in hospitalisations after an intervention to manage severe asthma. Eur Respir J, Mar;35(3):515-21, 2010.

SZWARCWALD CL, et al. Desigualdades socioeconômicas em saúde no Brasil: resultado da pesquisa Mundial de Saúde, 2003. Revista Brasileira de Saúde Materno Infantil, v. Supl1, p. 1122, 2005.

VICTORA CG, et al. Explaining trends in inequities: evidence from Brazilian child health studies. Lancet 356: 1093 - 1098, 2000.

VICTORA CG, et al. Lancet Brazil Series Working Group. Health conditions and health-policy innovations in Brazil: the way forward. Lancet, 377(9782):2042-53, 2011.

VICTORA CG et al. Maternal and child health in Brazil: progress and challenges. Lancet, 377(9780):1863-762011.

WORLD HEALTH ORGANIZATION. World Health Report 2002. Reducing risks, promoting healthy life. Geneva: WHO; 2002. 